\title{
Combining nanoimprint lithography and a molecular weight selective thermal reflow for the generation of mixed 3D structures
}

\author{
Arne Schleunitz ${ }^{\mathrm{a})}$ and Christian Spreu \\ Paul Scherrer Institut, Laboratory for Micro- and Nanotechnology, 5232 Villigen PSI, Switzerland \\ Marko Vogler and Hakan Atasoy \\ Micro Resist Technology GmbH, Koepenicker Str. 325, 12555 Berlin, Germany \\ Helmut Schift \\ Paul Scherrer Institut, Laboratory for Micro- and Nanotechnology, 5232 Villigen PSI, Switzerland
}

(Received 24 June 2011; accepted 30 August 2011; published 27 September 2011)

\begin{abstract}
Sloped and stepped 3D structures were added to surface-patterned resists using grey-scale electron beam lithography and thermal reflow. A poly(methyl methacrylate) resist with moderate initial molecular weight of $120 \mathrm{~kg} / \mathrm{mol}$ was chosen, which enabled processing with both nanoimprint and electron beam lithography. Using proper exposure doses, a molecular weight distribution was generated that allowed a selective thermal postprocessing of the exposed steps while the imprinted gratings on top of the resist were preserved. This allows fabricating mixed structures of microprisms surrounded by large-area nanogratings in the same resist layer. Working stamps were casted from the template pattern and subsequently replicated using thermal nanoimprint. As a possible application, backlight devices with arrays of light outcoupling prisms can be seen. (C) 2011 American Vacuum Society. [DOI: 10.1116/1.3643761]
\end{abstract}

\section{INTRODUCTION}

Mixed 3D structures are combined patterns at the microand nanometer scale with different sizes, shapes, depths, and lateral extension on the same substrate. Normally they are fabricated using the combinations of different processes in a sequential way. ${ }^{1-3}$ They are highly attractive for various applications in optics, fluidics, or biosciences, where a complex surface profile adds an improved functionality to elements or devices, e.g., if prisms have to be used for light coupling while the rest of the area has to be covered with a large-area binary grating for antireflection. ${ }^{4}$ For this, often a generic $3 \mathrm{D}$ patterning process has to be combined with more standardized lithography processes, which are routinely used to generate large-area quasibinary patterns, but are less qualified to create complex shapes as true 3D geometries. The generic processes for 3D patterning, however, are often not suitable for large areas or involve processing steps that can alter or destroy the original nanopatterns. Such a process is a thermal annealing, which is often used to smoothen out surface roughness or corrugations by reflow, ${ }^{5,6}$ or to generate new structures such as lenses and slopes. ${ }^{7,8}$

We have now combined nanoimprint lithography (NIL) and 3D electron beam lithography (EBL) with a thermal postprocessing in a way that the nanoimprinted structures were preserved while the shape of other exposed structures could be altered by reflow. This was possible because greyscale EBL, commonly used to generate 3D multilevel resist structures, enables a selective reflow due to a localized reduction in the glass transition temperature $\left(T_{g}\right)$ in the exposed areas. ${ }^{8}$ In this way, 3D patterns with footprints of a few micrometers are achieved, which can exhibit both nearly vertical sidewalls and inclined surfaces with angles up to

${ }^{a)}$ Electronic mail: arne.schleunitz@psi.ch $45^{\circ}$. In contrast to the research shown with EBL patterning alone, the successful combination of thermal reflow with structures patterned by NIL and EBL was possible by using a resist with a molecular weight suitable for both lithography techniques. $^{9-11}$ Since large-area imprints are subsequently complemented with specific 3D pattern showing sloped and vertical sidewalls, the process combination is especially interesting for the stamp manufacture of the complex master pattern needed for the high volume production of mixed 3D structures based on replication techniques.

The generation of mixed 3D resist structures based on the combination of generic processes is sketched in Fig. 1. First, a surface relief pattern is imprinted into a (thermoplastic) polymer film by standard thermal NIL (Ref. 12) using a nanostructured mold. Subsequently, the prepatterned polymer film is used as a positive resist in a grey-scale EBL exposure, where a multilevel resist contour is generated in the exposed areas after the wet development step due to the dose-dependent etch rate. Finally, the film with the superimposed imprinted and etched relief is subject to an annealing step at a temperature close to the resist's original $T_{g}$. This reflow at a moderate temperature leads to a selective transformation of the stepped sidewall profiles into smooth inclined slopes, while the embossed surface pattern stays unaltered.

\section{EXPERIMENT}

All mixed 3D structures have been fabricated in $1 \mu \mathrm{m}$ thick poly(methyl methacrylate) (PMMA) resist with $120 \mathrm{~kg} / \mathrm{mol}$, spin-coated on a dehydrated silicon substrate (size: $2 \times 2 \mathrm{~cm}^{2}$ ) and prebaked for $300 \mathrm{~s}$ at $175^{\circ} \mathrm{C}$ on a hot plate. The PMMA layer was prepatterned by thermal NIL using an equally sized silicon stamp into which a periodic line grating (width, height, and distance of cavities: $250 \mathrm{~nm}$ ), which was coated with a 


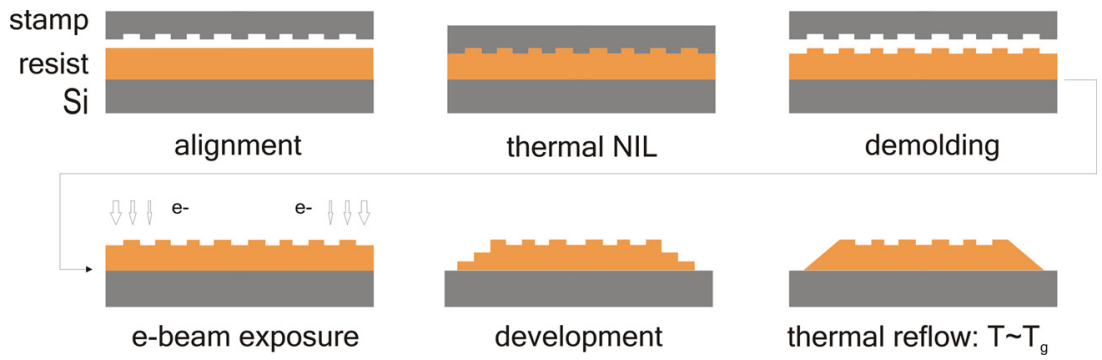

FIG. 1. (Color online) Process sequence based on three different generic processes subsequently applied for the generation of mixed 3D pattern: combining thermal NIL, grey-scale e-beam exposure, and reflow allows a selective transformation of stepped sidewalls into smooth slopes while imprinted surface pattern stays unaffected.

standard anti-sticking coating. The embossing was performed on a Jenoptik HEX03 NIL machine at $180^{\circ} \mathrm{C}$ for $600 \mathrm{~s}$ at around $15 \mathrm{MPa}$. The dose-modulated $\mathrm{EBL}$ was performed with a high energy electron beam (e beam) in a commercial pattern generator (Vistec EBPG 5000+ HR at $100 \mathrm{keV} / 1 \mathrm{nA}$ ). Exposed samples were developed for $30 \mathrm{~s}$ under slight agitation in a developer bath with undiluted methyl-isobutyl-ketone (MIBK) at $20^{\circ} \mathrm{C}$. After development, the samples were rinsed for $30 \mathrm{~s}$ in isopropyl alcohol and blow dried using nitrogen. The thermal postexposure treatment was performed on a hot plate under ambient conditions at $120^{\circ} \mathrm{C}$ for $15 \mathrm{~min}$. The reflow step was stopped by instant cooling when the sample was removed from the heater. All these parameters are similar to the process conditions used until now for $950 \mathrm{~kg} / \mathrm{mol}$ PMMA without imprint step. ${ }^{8,13,14}$

\section{RESULTS AND DISCUSSION}

For a successful process combination of thermal NIL, grey-scale EBL, and reflow, two prerequisites have to be fulfilled. First, the resist patterning is allowed by using both NIL and EBL. Second, a selective processing (melting) of the exposed structures is enabled while imprinted patterns stay unaltered. In our investigations presented here, we chose PMMA as resist material since it is a widely used standard resist for both NIL and EBL. Interestingly, in both cases the molecular weight typically has a significant influ-

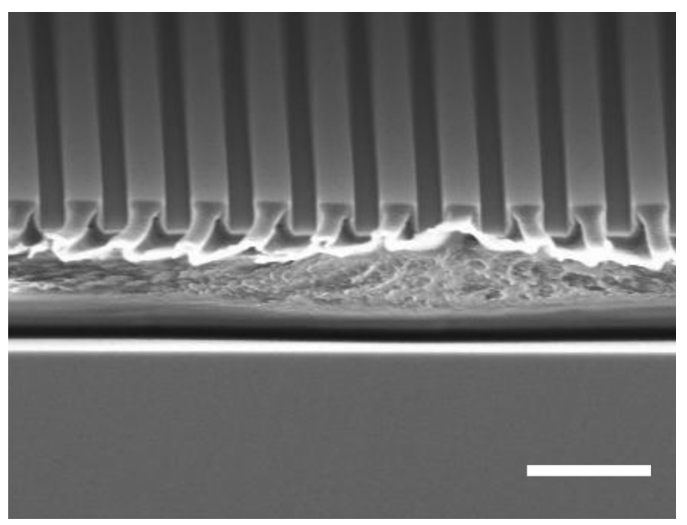

FIG. 2. Prepatterning of a $1 \mu \mathrm{m}$ thick PMMA layer with a molecular weight of $120 \mathrm{~kg} / \mathrm{mol}$ was accomplished by thermal NIL. The polymer layer on silicon is imprinted on a large area with a binary nanograting (height, width, and distance: $250 \mathrm{~nm}$ ). During sample cleaving, the resist is distorted and hence slightly detached from the substrate (scale bar: $1 \mu \mathrm{m}$ ). ence on the resolution capabilities, although in an opposed way: for thermal NIL, which is based on the spatial displacement of the resist material during imprint, a low viscosity of the heated resist is desired, which is accomplished by a rather low molecular weight of typically $25 \mathrm{~kg} / \mathrm{mol}$ or lower. For EBL, on the other hand, a high initial molecular weight of above $600 \mathrm{~kg} / \mathrm{mol}$ is advantageous due to the increased contrast during the development of exposed (reduced molecular weight $)^{10}$ and unexposed areas, i.e., an optimized difference in solubility upon development.

For a successful process combination, an appropriate molecular weight of the PMMA has to be used that meets the opposite material requirements for NIL and EBL application at the same time. We used a commercially available PMMA resist with a specific molecular weight of $120 \mathrm{~kg} / \mathrm{mol}$ provided by micro resist technology (Berlin, Germany). It was expected to be low enough to achieve viscous flow during imprint, but high enough to perform grey-scale EBL and eventually to facilitate a sufficient drop in $T_{g}$ for the exposed areas that selective reflow becomes possible.

The ability of the $120 \mathrm{~kg} / \mathrm{mol}$ PMMA to be replicated in the nanometer range was investigated by imprinting the $250 \mathrm{~nm}$ binary lin egrating onto the resist surface. Due to the relatively high thickness of $1 \mu \mathrm{m}$, which usually facilitates the cavity filling in contrast to thinner layers, a pattern replication was accomplished with a good fidelity. The result of the surface-patterned resist can be seen in Fig. 2. Due to the

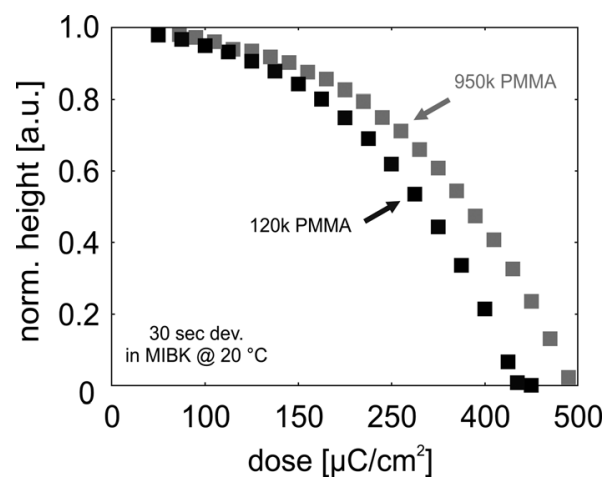

FIG. 3. Normalized dose-depth correlations (contrast curve) are plotted for $1 \mu \mathrm{m}$ thick PMMA with $120 \mathrm{~kg} / \mathrm{mol}$ (NIL resist) and $950 \mathrm{~kg} / \mathrm{mol}$ (standard EBL resist) on silicon. The e-beam exposure was performed at $100 \mathrm{keV} / 1 \mathrm{nA}$ and the development was accomplished in undiluted $\mathrm{MIBK}$ at $20^{\circ} \mathrm{C}$ for $30 \mathrm{~s}$. Since the contrast curves exhibit comparable slope characteristics, a greyscale EBL can also be applied to the NIL resist. 


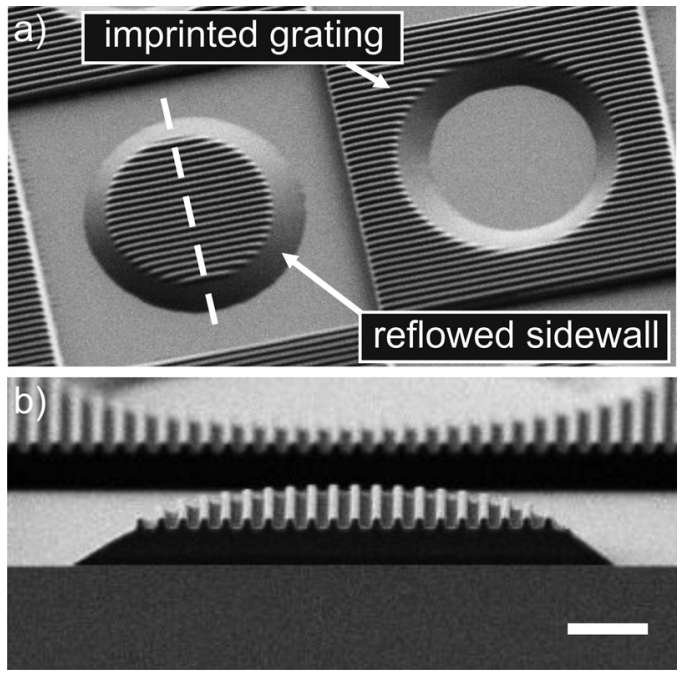

FIG. 4. Exemplary mixed 3D pattern was manufactured by combining NIL, grey-scale EBL, and thermal postprocessing (a). The PMMA structures consist of a binary $250 \mathrm{~nm}$ line grating on top of a $1 \mu \mathrm{m}$ thick resist with vertical and sloped sidewalls with an inclination $\sim 30^{\circ}$ (b) (scale bar: $2 \mu \mathrm{m}$ ).

polarity of the mold, i.e., recessed cavities with a net volume of less than 3\% compared to the available PMMA volume, the average resist height was only marginally reduced during the imprint procedure.

In order to further explore the capability of the $120 \mathrm{~kg} / \mathrm{mol}$ PMMA to be used as grey-scale EBL resist, the dose-depth correlation (contrast curve) was determined from the exposure of $200 \times 200 \mu \mathrm{m}^{2}$ rectangles at various doses. After the development, contact profilometry was used to measure the remaining resist heights. Figure 3 depicts the resulting normalized contrast curve and allows a comparison to the high molecular weight $950 \mathrm{~kg} / \mathrm{mol}$ PMMA that was used for grey-scale EBL in previous works. ${ }^{8,13,14}$ Although the dose to clear (complete removal of PMMA upon development) was found to be slightly decreased to $440 \mu \mathrm{C} / \mathrm{cm}^{2}$, the two comparable slope characteristics demonstrate the possibilities to apply a greyscale EBL to both resist types. This means that a patterning of the $120 \mathrm{~kg} / \mathrm{mol}$ PMMA resist can be accomplished by nanoimprint as well as grey-scale EBL, which fulfills the first of two prerequisites for the desired process combination.

With the above-described hybrid approach, exemplary mixed 3D structures were generated by performing a generic grey-scale EBL onto the $120 \mathrm{~kg} / \mathrm{mol}$ PMMA resist prepatterned with the $250 \mathrm{~nm}$ line grating. After development, the standard thermal treatment is applied to the resist patterns using a hot plate. Although the sample is generally heated for $15 \mathrm{~min}$ to $120^{\circ} \mathrm{C}$, only the e-beam exposed multilevel profile with a few steps is softened and transformed into continuous linear slopes. The imprinted surface pattern, however, stays unaltered upon the reflow step, which fulfills the second requirement for the process combination and is illustrated by the SEM micrographs in Fig. 4.

The demonstrated pattern selective shape transformation is based on the molecular weight dependence of thermal reflow. The grey-scale EBL of the PMMA leads to multilevel resist pattern with a locally reduced ${ }^{10}$ (number average) molecular weight $M_{n}$ below the $T_{g}$-relevant value of $10 \mathrm{~kg} / \mathrm{mol},{ }^{15}$ where differences in $T_{g}$ become significant and a pronounced decrease in viscosity during reflow is observed. Since the line grating was created by spatial displacement without a chemical modification, the $T_{g}$ of the surface structures is identical to the original $T_{g}$ of the $120 \mathrm{~kg} / \mathrm{mol}$ PMMA resist at $122{ }^{\circ} \mathrm{C}$. ${ }^{14}$ Consequently, the high resistance in the original material to deform prevents the replicated pattern from losing its shape during reflow. Interestingly, this applies for any kind of imprinted contour including 3D structure, which might be used to further enhance the structural variety in the future. Note that there is a slight distortion of the $3 \mathrm{D}$ pattern, i.e., the imprinted and EBL exposed patterns are not perfectly concentric after the reflow step, which might be caused by a relaxation of material stress induced during the thermal imprint process. This has to be further investigated in future research.

In addition to the thermally activated shape transformation, the surface tension driven self-perfection eliminates roughness within the sloped contours. This allows the generation of highly smooth but tilted surfaces especially suitable for optical applications. Such a structure is shown in Fig. 5. It consists of grey-scale exposed multistep elements which are integrated into an imprinted line grating. The selective melting enables the transformation of rough slopes into smooth prisms. Furthermore, corrugations within the slopes due to the overlay of imprinted with etched patterns are smoothed out, which assures light redirection with minimized diffraction loss, e.g., as needed by backlight device. $^{16}$
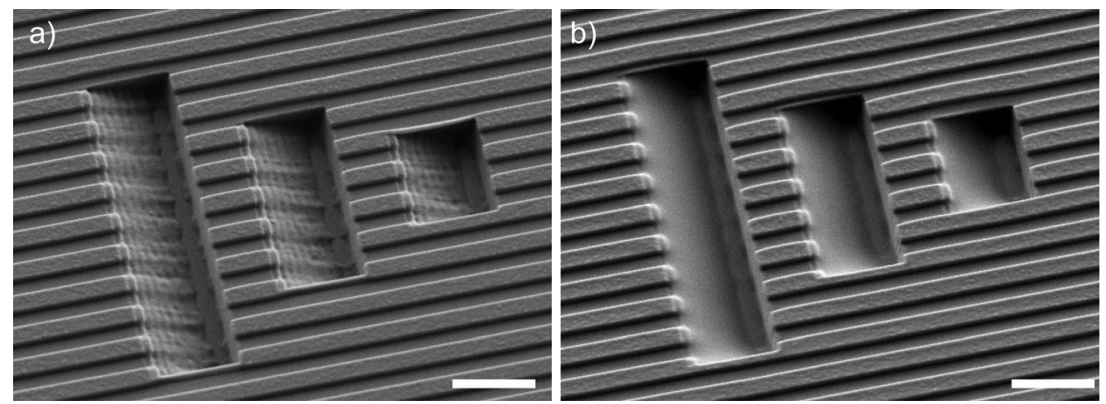

FIG. 5. SEM micrograph of an exemplary mixed 3D structure in PMMA resist intended for optical application for backlight display devices. The resist pattern is generated by grey-scale EBL onto an NIL prepatterned resist (a). The selective melting of exposed areas eventually integrates prisms with smooth surfaces into the imprinted line grating (b) (scale bar: $2 \mu \mathrm{m}$ ). 

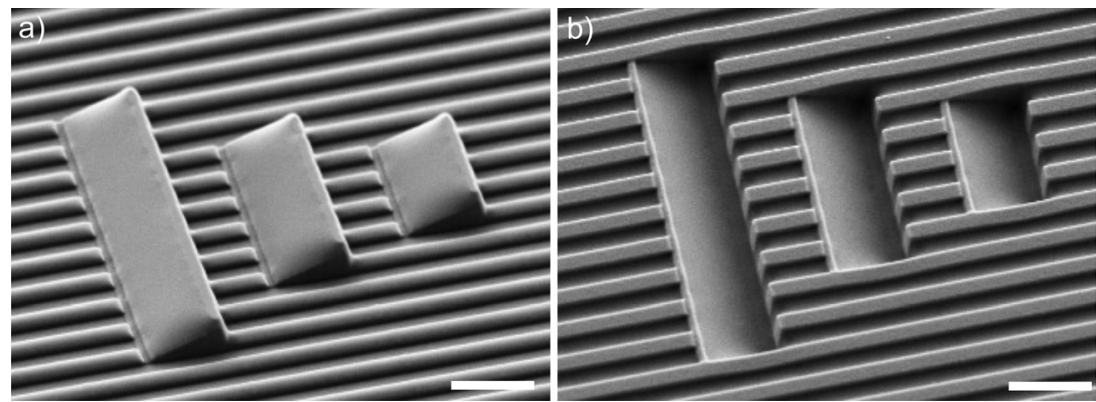

FIG. 6. SEM micrograph of an OrmoStamp mold (a), replicated from the PMMA master pattern, shows the inverse pattern polarity. Used as a working stamp, it allows a repeated generation of mixed 3D structure by replication techniques (b) (scale bar: $2 \mu \mathrm{m}$ ).

\section{CONCLUSION AND OUTLOOK}

Due to optimized process conditions, neither the EBL nor the thermal treatment affects the imprinted surface prepattern. This enhances current fabrication methods in many respects: complex resist shapes with features at different length scales are enabled and a micro-nano-integration for 3D pattern is simplified. Furthermore, since the generation of basic binary pattern is separated from extensive 3D machining, technical efforts and the overall processing complexities can be significantly reduced.

Targeting industrial applications where high production volumes are required, the presented example shows that NIL cannot only be considered as an enabling technique for the 3D manufacturing (production) due to its capabilities to replicate complex nonbinary pattern, but is also a suitable technology to address the technical challenges already in the phase of high quality master stamp fabrication and duplication. A key advantage of using NIL for the prepatterning of (EBL compatible) resist is the possibility to easily generate a large-area grating (e.g., for antireflection or wavelength filtering) by using appropriate stamp sizes, step-and-repeat processes or roll-embossing before complex $3 \mathrm{D}$ structures (e.g., backlight prisms) are only added to specific locations determined by the intended functionality. Once such structure with a complex 3D surface relief is generated, it can be transferred into a working stamp and used for direct replication. Figure 6 shows the replication of the mixed pattern into an OrmoStamp ${ }^{\circledR}$ layer on a glass substrate using the standard process described elsewhere. ${ }^{17}$ As an example for a one-step replication of mixed patterns, the working stamp was subsequently used to repeatedly replicate the mixed 3D pattern into $1 \mu \mathrm{m}$ thick thermoplastic resist mr-I T85 (micro resist technology, Berlin).

\section{ACKNOWLEDGMENTS}

The authors thank A. Schander, V. A. Guzenko, and C. David (PSI Villigen) for their help and valuable contributions. The research presented here was partially funded by Swiss Federal Office for Science and Education in the framework of the EC-funded project NaPANIL (Contract No. NMP 214249).

${ }^{1}$ X. Cheng and L. J. Guo, Microelectron. Eng. 71, 277 (2004).

${ }^{2}$ M. Tormen, F. Romanato, M. Altissimo, L. Businaro, P. Candeloro, and E. M. Di Fabrizio, J. Vac. Sci. Technol. B 22, 766 (2004).

${ }^{3}$ M. Tormen et al., Microelectron. Eng. 73-74, 535 (2004).

${ }^{4}$ M. B. Christiansen, J. Malgorzata Lopacinska, M. Havsteen Jakobsen, N. A. Mortensen, M. Dufva, and A. Kristensen, Opt. Express 17, 2722 (2009).

${ }^{5}$ Z. Yu, L. Chen, W. Wu, H. Ge, and S. Y. Chou, J. Vac. Sci. Technol. B 21, 2089 (2003).

${ }^{6}$ Y. Liang, P. Murphy, W. Li, and S. Y. Chou, Nanotechnology 20, 465305 (2009).

${ }^{7}$ H. Schift, C. Spreu, A. Schleunitz, and J. J. Lee, Microelectron. Eng. 88, 87 (2011).

${ }^{8}$ A. Schleunitz and H. Schift, J. Micromech. Microeng. 20, 095002 (2010).

${ }^{9}$ H.-C. Scheer, N. Bogdanski, M. Wissen, and S. Möllenbeck, Microelectron. Eng. 85, 890 (2008).

${ }^{10}$ E. A. Dobisz, S. L. Brandow, R. Bass, and J. Mitterender, J. Vac. Sci. Technol. B 18, 107 (2000).

${ }^{11}$ H. R. Keymeulen, A. Diaz, H. H. Solak, C. David, F. Pfeiffer, B. D. Patterson, J. F. van der Veen, M. P. Stoykovich, and Paul F. Nealey, J. Appl. Phys. 102, 013528 (2007).

${ }^{12}$ H. Schift, J. Vac. Sci. Technol. B 26, 458 (2008).

${ }^{13}$ A. Schleunitz and H. Schift, Microelectron. Eng. 88, 2736 (2011).

${ }^{14}$ A. Schleunitz, V. A. Guzenko, A. Schander, M. Vogler, and H. Schift, J. Vac. Sci. Technol. B 29, 06F302 (2011).

${ }^{15}$ K. Ute, N. Miyatake, and K. Hatada, Polymer 36, 1415 (1995).

${ }^{16}$ T. Mäkelä, T. Haatainen, J. Ahopelto, J. Oravasaari, T. Tuohioja, and K. Rinko, Proceedings of the Conference on Nanoimprint and Nanoprint Technology (NNT), Øresund, Copenhagen, Denmark, 13-15 October 2010 (unpublished).

${ }^{17}$ H. Schift, C. Spreu, M. Saidani, M. Bednarzik, J. Gobrecht, A. Klukowska, F. Reuther, G. Gruetzner, and H. H. Solak, J. Vac. Sci. Technol. B 27, 2846 (2009). 